OPEN ACCESS

Edited by:

Peng Xiao,

Sun Yat-sen University, China

Reviewed by:

Elisabetta Pilotto,

University Hospital of Padua, Italy

Xiaoyan Ding

Sun Yat-sen University, China

John Nickerson,

Emory University, United States

*Correspondence:

Kari V. Vienola

kvienola@ucdavis.edu

Specialty section:

This article was submitted to

Ophthalmology,

a section of the journal

Frontiers in Medicine

Received: 01 September 2021 Accepted: 08 November 2021 Published: 09 December 2021

Citation:

Vienola KV, Dansingani KK, Eller AW, Martel JN, Snyder VC and Rossi EA (2021) Multimodal Imaging of Torpedo Maculopathy With Fluorescence Adaptive Optics Imaging of Individual Retinal Pigmented Epithelial Cells.

Front. Med. 8:769308.

doi: 10.3389/fmed.2021.769308

\section{Multimodal Imaging of Torpedo Maculopathy With Fluorescence Adaptive Optics Imaging of Individual Retinal Pigmented Epithelial Cells}

\author{
Kari V. Vienola ${ }^{1 *}$, Kunal K. Dansingani ${ }^{1}$, Andrew W. Eller ${ }^{1}$, Joseph N. Martel ${ }^{1}$, \\ Valerie C. Snyder ${ }^{1}$ and Ethan A. Rossi ${ }^{1,2,3}$
}

${ }^{1}$ Department of Ophthalmology, University of Pittsburgh, Pittsburgh, PA, United States, ${ }^{2}$ Department of Bioengineering, University of Pittsburgh Swanson School of Engineering, Pittsburgh, PA, United States, ${ }^{3}$ McGowan Institute for Regenerative Medicine, University of Pittsburgh, Pittsburgh, PA, United States

Torpedo maculopathy (TM) is a rare congenital defect of the retinal pigment epithelium (RPE). The RPE is often evaluated clinically using fundus autofluorescence (AF), a technique that visualizes RPE structure at the tissue level from the intrinsic AF of RPE fluorophores. TM lesions typically emit little or no AF, but this macroscopic assessment is unable to resolve the RPE cells, leaving the organization of the RPE cell mosaic in TM unknown. We used fluorescence adaptive optics scanning laser ophthalmoscopy (AOSLO) to show here for the first time the microscopic cellular-level structural alterations to the RPE cell mosaic in TM that underlie the tissue-level changes seen in conventional clinical imaging. We evaluated two patients with TM using conventional clinical imaging techniques and adaptive optics (AO) infrared autofluorescence (IRAF) in AOSLO. Confocal AOSLO revealed relatively normal cones outside the TM lesion but altered cone appearance within it and along its margins in both patients. We quantified cone topography and RPE cell morphometry from the fovea to the margin of the lesion in case 1 and found cone density to be within the normal range across the locations imaged. However, RPE morphometric analysis revealed disrupted RPE cells outside the margin of the lesion; the mean RPE cell area was greater than two standard deviations above the normative range up to approximately $1.5 \mathrm{~mm}$ from the lesion margin. Similar morphometric changes were seen to individual RPE cells in case 2. Multi-modal imaging with AOSLO reveals that RPE cells are abnormal in TM well beyond the margins of the characteristic TM lesion boundary defined with conventional clinical imaging. Since the TM fovea appears to be fully formed, with normal cone packing, it is possible that the congenital RPE defect in TM occurs relatively late in retinal development. This work demonstrates how cellular level imaging of the RPE can provide new insight into RPE pathologies, particularly for rare conditions such as TM.

Keywords: torpedo maculopathy, retinal pigment epithelium, autofluorescence, adaptive optic systems, scanning laser ophthalmoscopy, fluorescence imaging 


\section{INTRODUCTION}

Torpedo maculopathy (TM) is characterized by the presence of a well-circumscribed lesion of the macula featuring elements of hyperpigmentation and atrophy at the level of the retinal pigment epithelium (RPE) and named for its typical shape and orientation with respect to the fovea $(1,2)$. TM appears to be congenital, nonprogressive, and uncommon. Prevalence has been estimated to be 2 per 100,000 (3) with fewer than 100 cases reported as of 2019 (4). Early conjectures about the most appropriate classification included a subclass of congenital nevus of the RPE, or a form of congenital hypertrophy of the RPE $(5,6)$, but these descriptions pre-date optical coherence tomography (OCT).

In TM, OCT sometimes reveals neurosensory retinal layers detached from the RPE, forming a cavity $(7,8)$. Based on this, Wong et al. classified TM into Types I and II, the latter including neurosensory detachment with subretinal cavitation (9). Often the OCT B-scans show increased light penetration to the choroid with minimal fundus autofluorescence (FAF) signal originating from the lesion $(2,10)$. Functional testing with visual fields (11) and microperimetry (9) has shown reduced sensitivity in the lesion. Collective responses from electroretinogram (ERG) exams performed on patients with TM have not shown any abnormalities (12). However, multifocal ERG has shown amplitude reduction with potential latency between pathological and healthy retinal areas of the same eye $(12,13)$.

In recent years, OCT angiography (OCTA) has been used to evaluate flow profiles in the choriocapillaris and/or choroid in the lesions. Most of these studies have reported decreased flow in the choriocapillaris with vascular alterations at the site of the lesion (11, 14-16). Giannakaki-Zimmermann et al. (17) concluded that attenuation of OCTA signal in choriocapillaris occurs with Type I as well, when there is no subretinal cavitation on structural OCT. Although one publication reported an increase in choroidal vascular density, no explanation was offered as to how this was measured and closer inspection of the key figure in the paper shows reduced choriocapillaris flow density in the lesion, compared with areas outside the lesion (18).

Adaptive optics ophthalmoscopy (AOO) permits cellular level imaging of the retina $(19,20)$. Recently, Hugo et al. used a commercially available flood-illumination adaptive optics (FIAO) fundus camera to evaluate TM patients and showed decreased cone density in the lesion compared to the healthy retina, suggestive of cone loss (21). Similar findings were reported by Lambart et al. (22) however, they hypothesized that cones were axially displaced rather than lost. Here, we present two new patients with TM who were evaluated using standard clinical imaging tools and AOO, including a commercial FIAO retinal camera and a custom fluorescence adaptive optics scanning laser ophthalmoscope (AOSLO). Compared to FIAO, AOSLO can achieve higher resolution for "reflectance" (i.e., backscattered) light imaging due to its ability to reject out-of-focus light (confocality). Our AOSLO is also equipped with a near-infrared autofluorescence detection channel that we used here to define the morphological alterations to the RPE cell mosaic associated with TM. To our knowledge, this is the first time that RPE
TABLE 1 | Optical coherence tomography imaging parameters.

\begin{tabular}{lccc}
\hline OCT parameters & Patient 1 & \multicolumn{2}{c}{ Patient 2 } \\
\cline { 2 - 4 } & $\mathbf{2 0 1 9}$ & $\mathbf{2 0 1 7}$ & $\mathbf{2 0 1 9}$ \\
\hline Size X & 1,536 pixels & 768 pixels & 1,024 pixels \\
& $(9.8 \mathrm{~mm})$ & $(9.1 \mathrm{~mm})$ & $(6.1 \mathrm{~mm})$ \\
Size Z & 496 pixels & 496 pixels & 496 pixels \\
& $(1.9 \mathrm{~mm})$ & $(1.9 \mathrm{~mm})$ & $(1.9 \mathrm{~mm})$ \\
Scaling X & $6.41 \mu \mathrm{m} /$ pixel & $11.79 \mu \mathrm{m} /$ pixel & $6.00 \mu \mathrm{m} /$ pixel \\
Scaling Z & $3.87 \mu \mathrm{m} /$ pixel & $3.87 \mu \mathrm{m} /$ pixel & $3.87 \mu \mathrm{m} /$ pixel \\
ART Mode & ON $(16 \mathrm{averaged})$ & ON $(9$ averaged $)$ & ON $(15 \mathrm{averaged})$ \\
Quality & $38 \mathrm{~dB}$ & $42 \mathrm{~dB}$ & $26 \mathrm{~dB}$ \\
EDI Mode & ON & OFF & ON \\
Number Of & 49 & 61 & 49 \\
B-Scans & & & \\
Pattern Size & $30^{\circ} \times 10^{\circ}$ & $30^{\circ} \times 25^{\circ}$ & $20^{\circ} \times 20^{\circ}$ \\
& $(9.8 \times 3.3 \mathrm{~mm})$ & $(9.1 \times 7.5 \mathrm{~mm})$ & $(6.1 \times 6.1 \mathrm{~mm})$ \\
Distance between & $68 \mu \mathrm{m}$ & $126 \mu \mathrm{m}$ & $128 \mu \mathrm{m}$ \\
B-Scans & & & \\
\hline
\end{tabular}

morphology has been examined and quantified in vivo in TM patients using any modality.

Given the well-circumscribed nature of TM, our hypothesis was that the transition from abnormal to normal cellular mosaics would be relatively abrupt, with abnormalities confined to the immediate vicinity of the lesion. Further, due to the nonprogressive clinical course, we hypothesized that the demarcation between abnormal and normal photoreceptor and RPE mosaics would be approximately aligned, without one appearing to lead or lag the other in terms of distance from the lesion border.

\section{METHODS}

Clinical imaging included color fundus photography (Topcon/Canon), SLO/OCT (Spectralis, Heidelberg, Germany), and a flood-illumination adaptive optics camera (rtx1-e, Imagine Eyes, France). Detailed OCT imaging parameters are presented in Table 1. Microperimetry (MAIA, CenterVue S.p.A, Padova, Italy) was performed using both the macular test and with custom case-specific test patterns that we used to evaluate light sensitivity across the lesions. Before the testing started, optic nerve head was used to calibrate the measurement and for normal control data we used the normative database of the manufacturer. For patient 1, OCTA was also performed (Optovue, Fremont, CA, USA) using an $8 \times 8 \mathrm{~mm}$ scan area.

Adaptive optics scanning laser ophthalmoscopy imaging was carried out using a system that has been described in detail (23). Briefly, two imaging channels were used for simultaneous confocal reflectance and AO-IRAF imaging. A $795 \mathrm{~nm}$ super-luminescent diode was used for simultaneous confocal illumination and AO-IRAF excitation, with AF emission detected between 814 and $850 \mathrm{~nm}$ using a double-stacked bandpass filter (FF01-832/37, Semrock, USA). To ensure that no excitation light leaked into the AF detection channel, doublestacked filters were used in the $795 \mathrm{~nm}$ illumination path 


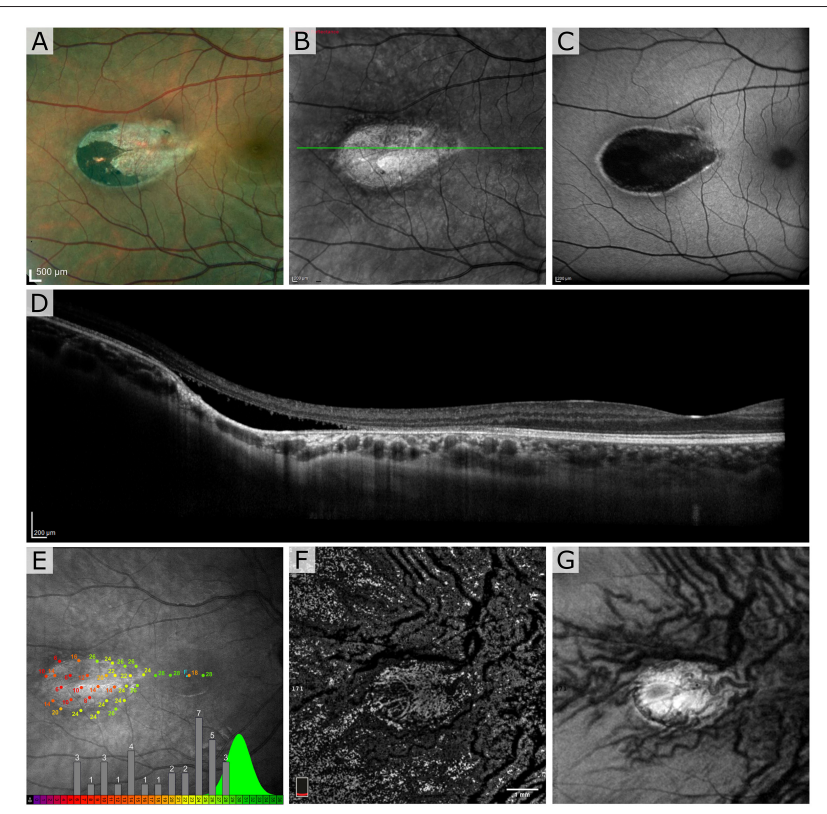

FIGURE 1 | Patient 1 imaged with multimodal clinical imaging. (A) Fundus images show a distinct torpedo-shaped lesion at the temporal macular region with the red box indicating the areas for the scanning laser ophthalmoscopy (SLO) and blue autofluorescence (BAF) images. (B,C) SLO reflectance and BAF image of the lesion showing the lesion very reflective in the SLO image and exhibiting marked hypo-AF signal at the lesion with a rim of hyper-AF. The green line in (B) indicates the B-scan location. (D) Optical coherence tomography (OCT) B-scan through the lesion. (E) Microperimetry test showing normal sensitivity in the fovea and severely decreased sensitivity at lesion with overall sensitivity well outside the normal distribution. The green distribution represents reference data from normal subjects. OCT en face angiogram (F) projection and corresponding structural image (G) taken with the OptoVue using a slab thickness of $70 \mu \mathrm{m}$ about $150 \mu \mathrm{m}$ below the retinal pigment epithelium (RPE).
(ET775/50x, Chroma, Bellows Falls VT, USA) to block any spontaneous emission in longer bands. The adaptive optics subsystem used a $909 \mathrm{~nm}$ laser diode beacon to detect the ocular aberrations and correct them in closed-loop mode with a deformable mirror. Imaging data were acquired at $30 \mathrm{~Hz}$ across a $1.5^{\circ} \times 1.5^{\circ}$ field of view for a duration of $60-70 \mathrm{~s}$.

The confocal images were used to co-register the weak signals in the AO-IRAF images using custom strip-based image registration software (24), permitting averaging to increase the signal-to-noise ratio in the AO-IRAF images. For the cell quantification from the AO-IRAF images, a semi-automated algorithm was used (25).

Patients were recruited through the clinics of the UPMC Eye Center. Written informed consent was obtained from both subjects following an explanation of experimental procedures and risks both verbally and in writing. All experiments were approved by the University of Pittsburgh Institutional Review Board and adhered to the tenets of the Declaration of Helsinki. To ensure safe imaging, all light levels were kept below the ANSI laser safety limits (26) and were calculated in accordance with best practices for multi-wavelength ophthalmic imaging (27).

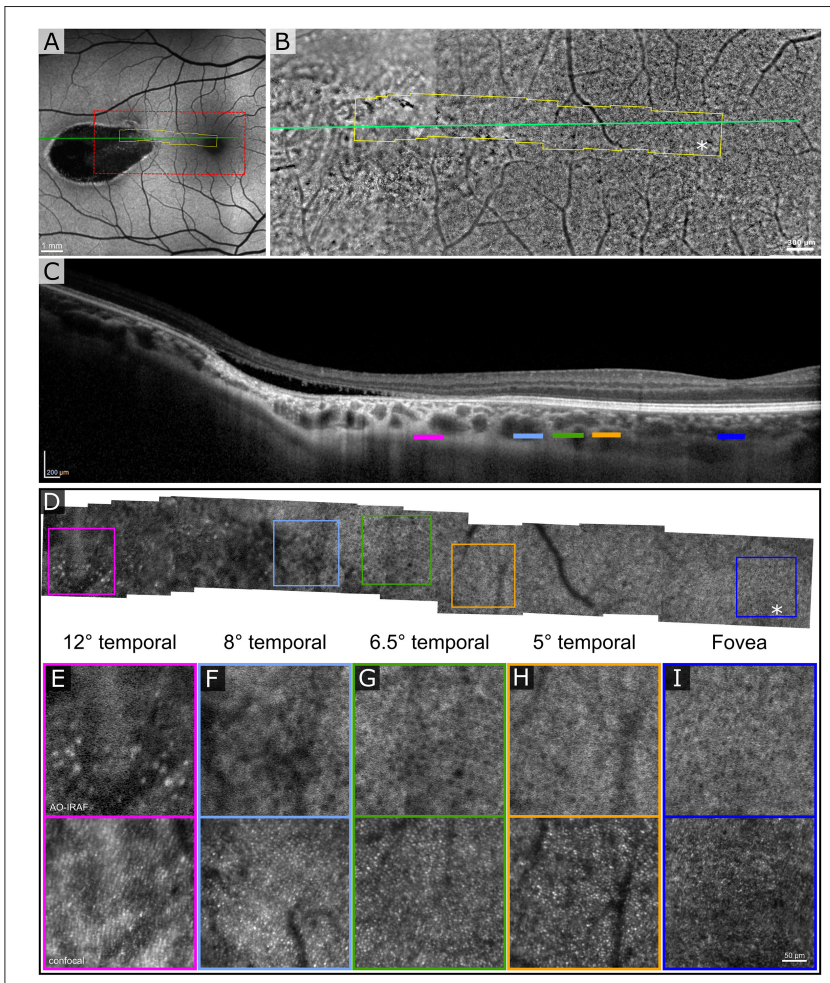

FIGURE 2 | Adaptive optics (AO) assisted imaging of patient 1. (A) Blue autofluorescence image showing the location of the lesion with red dashed box indicating the area imaged with the flood-illumination adaptive optics (FIAO) retinal camera and green line showing the location of the OCT scan. (B) FIAO montage showing typical photoreceptor mosaic in the region of the fovea. Near the lesion, the cone signature becomes irregular and cannot be distinguished anymore. The yellow area indicates the area that was imaged with the AOSLO. (C) OCT B-scan showing the cavity, indicating Type II torpedo maculopathy. Different color bars indicate roughly the location where AO-IRAF images where obtained. (D) AO-IRAF montage showing typical RPE cell mosaic. The cell size increases when moving to higher eccentricities and when reaching the lesion border, the mosaic eventually disappears. (E-I) Zoomed images of the regions of interest from the montage. Hypo-fluorescent spots are seen in (E) suggesting fluorophore accumulation in the lesion itself. Bottom row showing corresponding confocal image from the areas (E-I).

\section{RESULTS}

Patient 1, a woman in her early 30 s was found to have a pigmented lesion in her right eye during pre-screening for laser refractive surgery that was diagnosed later as TM. Best corrected visual acuity (BCVA) was 20/15 in each eye. The patient had no history of retinal laser nor injections prior to our imaging. Patient 2 was a woman in her early 20s diagnosed with TM. Her BCVA was 20/20 in each eye. The left eye had received a single intravitreal bevacizumab injection 2 years earlier for neovascularization within the lesion. Her angiography images have been published previously (28).

The first patient exhibited a typical torpedo-shaped lesion (Figures 1A-C) in the temporal macula with neurosensory detachment. Microperimetry showed decreased sensitivity in the lesion in both patients but in patient 1 with subretinal 
cavitation (Figure 1D), the expected zero sensitivity did not occur, suggesting some visual function remaining (Figure 1E). In the OCTA, image slices taken from approximately $150 \mu \mathrm{m}$ below the RPE showed some dense vascularization (Figures 1F,G) and what appears to be false positive flow signal temporal to the lesion. Figure $\mathbf{2 A}$ shows the blue autofluorescence image with areas marked where the $\mathrm{B}$-scan and flood AO imaging was done. The cone mosaic in the flood AO (Figure 2B) shows typical cone mosaic with structure disappearing with eccentricity when approaching the lesion (yellow box indicating the AOIRAF imaging area). This is consistent with the structural information on the B-scan (Figure 2C), showing the cavity fully starting at the edge of the AO-IRAF montage (Figure 2D). AO-IRAF showed the RPE mosaic (Figures 2E-I) visible from the fovea up to the margin of the lesion with an expected increase in cell size with increasing eccentricity. At the margin of the lesion, we observed heterogeneity in cell sizes as well as a decrease in the fluorescence signal corresponding to areas larger than individual RPE cells. Within the lesion, few individual RPE cells were seen on AO-IRAF and we observed several hyper-fluorescent spots with a diameter of $15-20 \mu \mathrm{m}$ (Figure 2E).

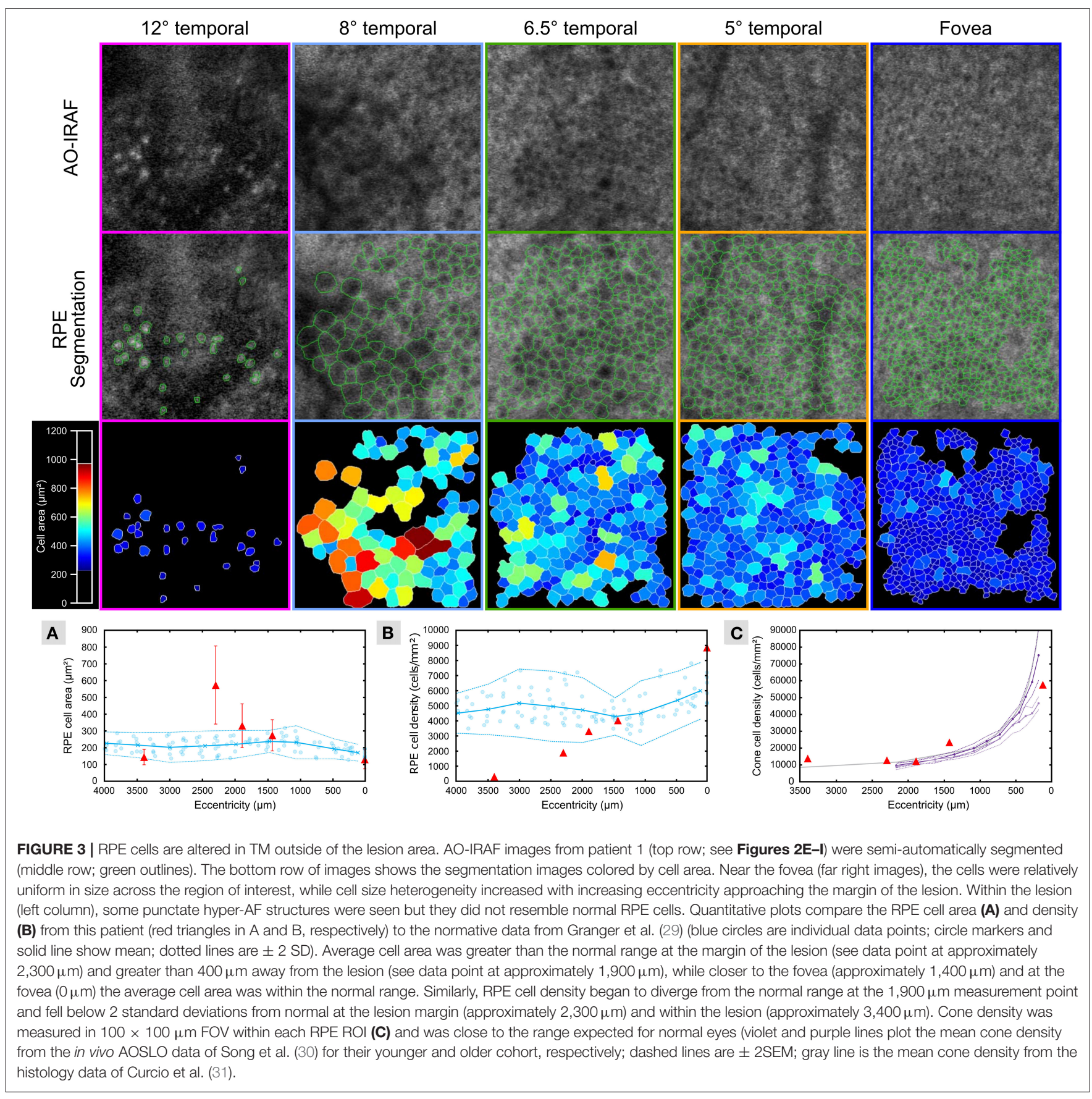


Figure 3 shows the segmentation of RPE cells from the different eccentricities (from 0 to $3,400 \mu \mathrm{m}$ ) from patient 1 . Average cell area (Figure 3A) was greater than the normal range [data from Granger et al. (29)] at the margin of the lesion (see data point at approximately $2,300 \mu \mathrm{m}$ ) and greater than 400 microns away from the lesion (see data point at approximately $1,900 \mu \mathrm{m}$ ), while closer to the fovea (approximately $1,400 \mu \mathrm{m}$ ); and at the fovea $(0 \mu \mathrm{m})$, the average cell area was within the normal range. The RPE cell density (Figure 3B) deviated from the normative data at the 1,900 microns measurement point and fell below 2 standard deviations from normal at the lesion margin (approximately 2,300 $\mu \mathrm{m}$ ) and within the lesion (approximately $3,400 \mu \mathrm{m})$. The cone density was measured in $100 \times 100 \mu \mathrm{m}$ FOV within each RPE ROI (Figure 3C) and was close to the range expected for normal eyes.

Since the second patient (Figure 4) was treated with intravitreal injection, we have shown the SLO fundus image, and an OCT B-scan taken prior to the injection in Figures 4A,B. A characteristic torpedo shaped lesion with irregular margins on its temporal aspect is seen in Figure 4C. Standard clinical FAF imaging shows reduced AF signal (Figures 4D-F) from the lesion area and substantial light penetration to the choroid on the OCT B-scan (Figure 4H) suggesting extensive disruption to the RPE. As in patient 1 , microperimetry in patient 2 showed reduced sensitivity within the lesion but it was not reduced to same extent seen in patient 1 (Figure 4D).

Figure 5A shows the clinical SLO image with red rectangle indicating the area imaged with flood-illumination $\mathrm{AO}$ (Figure 5B) and green line showing the location of the B-scan
(Figure 5C). Despite a very low AO-IRAF signal in some areas (Figure 5E), the cone mosaic was well defined and clearly visible at many locations within the lesion on FIAO (Figure 5B) and confocal AOSLO (Figure 5D). AO-IRAF did now show the typical RPE mosaic within the lesion but trace signatures of the mosaic and RPE cells are seen in the bottom left corner of the montage just outside the margins of the TM lesion as well as at the right edge of the AO-IRAF montage.

\section{DISCUSSION}

Herein, we show the in vivo morphology of both the cone and RPE cell mosaics in TM for the first time. These findings suggest that outside the TM lesion, RPE morphometry is relatively normal. However, near the margin of the lesion, we see marked changes in RPE morphometry characteristic of a disrupted cell mosaic. Within the TM lesion, we observed differences between the two cases that may reflect differences between type I and type II lesions. In patient 1 (Figures 1-3), with a type II lesion (neurosensory detachment), we saw reduced AO-IRAF compared to the normal appearing areas outside the lesion but there was still well-defined structure within the lesion such as several areas of hyper-autofluorescence similar in size to individual RPE cells. It is possible that these hyperautofluorescent areas represent RPE cells that have been altered due to the TM and accumulated additional NIR fluorophores. Another possible explanation of the hyperautofluorescence in the lesion borders could be RPE rounding/stacking but we did not
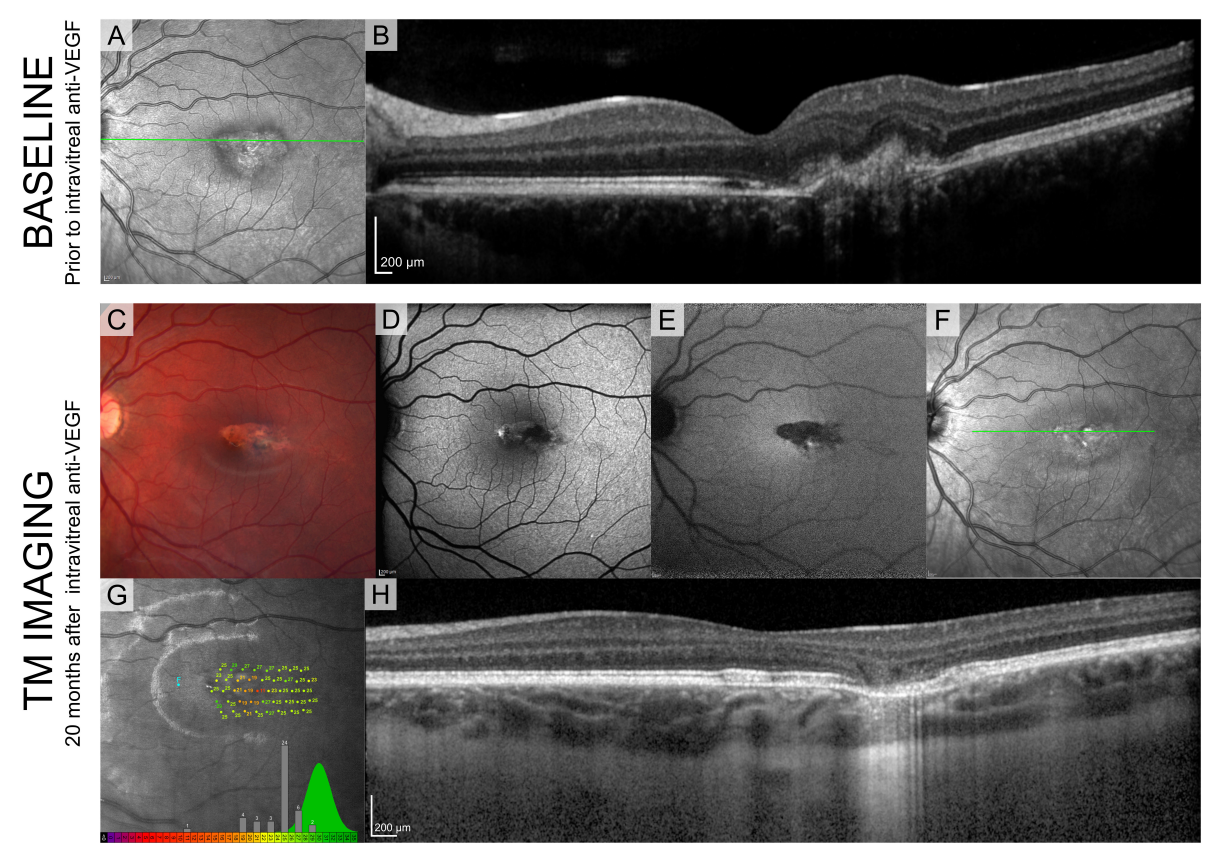

FIGURE 4 | Standard clinical imaging of patient 2. (A,B) SLO reflectance image and OCT B-scan taken from the location marked with a green line before the injection. (C) Fundus photograph 20 months after the injection. (D-F) SLO images from Spectralis (BAF, IRAF, reflectance) showing reduced FAF signal from the lesion. (G) Sensitivity estimates from MAIA show decreased values but compared to patient 1 they are substantially higher, suggesting intact visual sensitivity within the lesion. Green distribution represents normative data from the manufacturer's database. (H) OCT B-scan taken from the location marked with a green line in (D). 

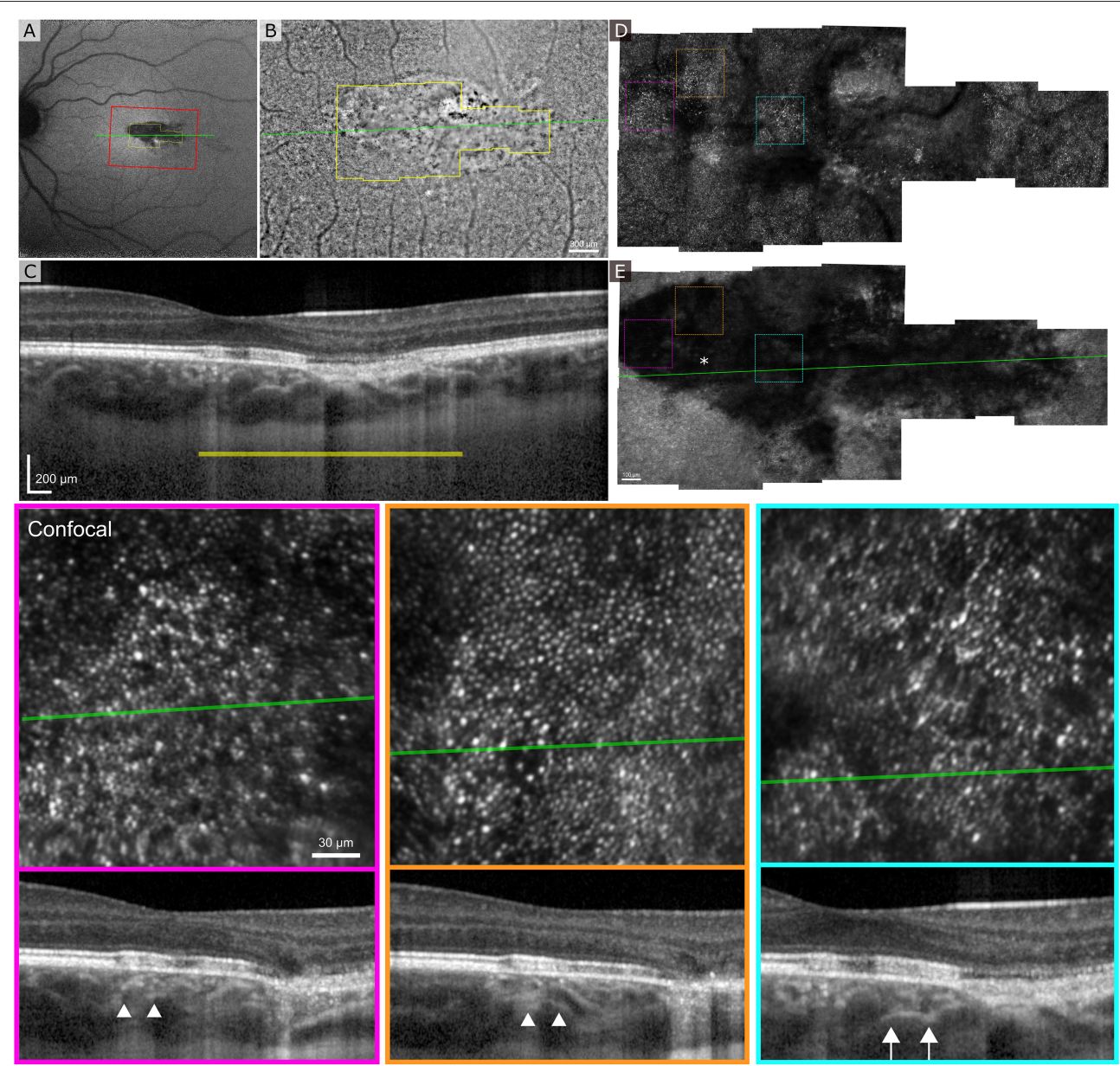

FIGURE 5 | Adaptive optics ophthalmoscopy imaging of the patient 2. (A) A clinical SLO reflectance image showing the location of the FIAO montage with red box and OCT B-scan with a green line going through the lesion. (B) FIAO montage showing typical photoreceptor mosaic outside the lesion. The yellow outline indicates the area imaged using AOSLO, with yellow bar showing the FIAO image location in the OCT B-scan. (C) B-scan showing extensive damage at the RPE layer with slightly increased light penetration to the choroid. (D) Confocal AOSLO montage of the lesion area. Compared to FIAO, the cone mosaic is sharper and has more contrast due to confocality and rejection of out-of-focus light. Even within the lesion patches of photoreceptor mosaic are seen. (E) AO-IRAF shows highly reduced FAF from the lesion throughout, even within areas where the photoreceptor mosaic seemed healthy, suggesting that the RPE underlying the photoreceptors is damaged. The colored dashed squares mark the location of the zoomed in areas at the bottom with colors indicating the areas. The B-scan locations are marked with a green line and in the B-scans the white arrow heads show the edges of the zoomed in area. The location of the fovea is marked with an asterisk in (E).

see evidence of this when we examined the B-scan stack going through the lesion, particularly the hyperAF areas.

In the second case, there was very little well-defined RPE structure within the TM lesion; though some areas of hyper- and hypo-autofluorescence were seen, no hyper-AF structures were seen that had a similar appearance to what was seen in the first case. Since the photoreceptor mosaic in the confocal channel was well-resolved we do not think that this is an artifact due to poor image quality but rather is reflective of the distribution of fluorophores within the RPE.

Compared to other studies that have implemented commercial flood-illumination adaptive optics retinal cameras in their studies, the confocal reflectance images show similar morphology with no clear photoreceptor mosaic visible $(21,22)$. Most likely the photoreceptors are there but are misaligned resulting in an altered reflectance signal.
Interestingly, some retinal sensitivity was preserved within the lesion bounds in both patients. This was unsurprising in patient 2, with the type I TM lesion, since it appeared both clinically and on AO-IRAF that sufficient RPE was present to support some photoreceptor survival and function. It was more surprising to see some evidence of photoreceptor function in patient 1 , in whom the RPE appeared macroscopically and on AO-IRAF to be mostly obliterated. Given that eyes with complete and chronic RPE atrophy often exhibit severe outer retinal disruption, this observation led us to speculate that neurosensory detachment from a pathological RPE might have been relatively protective to the photoreceptors. It also caused us to question whether TM is congenital, for if so, the observations suggest that it must arise relatively late in development, after outer retinal development and photoreceptor packing are completed. Considering our sensitivity losses in comparison to the literature, it appears that 
sensitivity losses in TM vary across a spectrum ranging from little to mild losses, such as the case of patient 3 with Wong et al. (9) to moderate losses such as seen in our case 2 to the more severe losses accompanied by cavitation such as in our case 1 and Wong's patient 4 .

As patient 2 underwent a single intravitreal bevacizumab injection prior to being imaged in our study, it is possible that that previous neovascularization might have altered the structure of the RPE. However, based on the literature (3, 14 ), it appears relatively common to have neovascularization on this rare condition, especially with type I. It is possible that there are some different alterations to the retina in TM with and without neovascularization. Without additional data, it is hard to interpret the level of influence on the AF signal itself.

Although the sample size for this descriptive study was limited by the low prevalence of TM, we were able to image both variants, type I and II, in detail. Clinically, TM can present with varying appearance and new possible lesion subtypes (32) may still be discovered such as those presenting with vitelliform material (33). Near infrared autofluorescence imaging using adaptive optics is an exciting new field and more quantitative results are possible in future studies.

\section{CONCLUSION}

Contrary to our expectation that the transition from abnormal to normal cellular mosaics would be relatively abrupt, with abnormalities confined to the immediate vicinity of the lesion, we found that RPE morphometric alterations extended well beyond the bounds of the clinically defined TM lesion. Additionally, the demarcation between abnormal and normal cone and RPE mosaics were not aligned, as cone density appeared to fall within the normal range at each of the locations where RPE cells were quantified in case 1, even though RPE cell morphometry was abnormal in several of these locations. The fluorescence signal that we detect with adaptive optics ophthalmoscopy can show alterations in the RPE when the structural cone images still appear normal. This was shown here in the case of TM patients, but this methodology can be adapted to various diseases and when accompanied by complimentary imaging modalities such as OCT, is a promising tool for clinicians to study the health of the retina.

\section{REFERENCES}

1. Teitelbaum BA, Hachey DL, Messner LV. Torpedo maculopathy. J. Am. Optom. Assoc. (1997) 68:373-6

2. Golchet PR, Jampol LM, Mathura JR, Daily MJ. Torpedo maculopathy. Br J Ophthalmol. (2010) 94:302-6. doi: 10.1136/bjo.2009.162669

3. Shirley K, O’Neill M, Gamble R, Ramsey A, McLoone E. Torpedo maculopathy: disease spectrum and associated choroidal neovascularisation in a paediatric population. Eye. (2018) 32:1315-20. doi: 10.1038/s41433-018-0074-7

\section{DATA AVAILABILITY STATEMENT}

The raw data supporting the conclusions of this article will be made available by the authors, without undue reservation.

\section{ETHICS STATEMENT}

The studies involving human participants were reviewed and approved by University of Pittsburgh Institutional Review Board. The patients/participants provided their written informed consent to participate in this study.

\section{AUTHOR CONTRIBUTIONS}

ER and KV conceived and conducted the experiment(s) and also drafted the manuscript. ER, KD, and KV analyzed and interpreted the results. VS conducted the clinical imaging. $\mathrm{JM}$ and AE critically revised the manuscript for important intellectual content. All co-authors carefully reviewed the final manuscript.

\section{FUNDING}

This research was supported by departmental startup funds from the University of Pittsburgh to ER. This work was also supported by the NIH CORE Grant P30 EY08098 to the University of Pittsburgh, Department of Ophthalmology, the Eye and Ear Foundation of Pittsburgh, NVIDIA GPU Grant Program and from an unrestricted grant from Research to Prevent Blindness, New York, NY, USA.

\section{ACKNOWLEDGMENTS}

The authors would like to thank Austin Roorda for sharing their AOSLO software, Pavan Tiruveedhula for electronics fabrication and software guidance and support, Jeff Speakman and Andrew Holmes for manufacturing custom mechanical components, and finally Jie Zhang, for sharing his original AOSLO optical design.

\section{SUPPLEMENTARY MATERIAL}

The Supplementary Material for this article can be found online at: https://www.frontiersin.org/articles/10.3389/fmed. 2021.769308/full\#supplementary-material

4. Williams PJ, Salek S, Prinzi RA, Bergstrom C, Hubbard GB. Distribution patterns of torpedo maculopathy: further evidence of a congenital retinal nerve fiber layer-driven etiology. Saudi J Ophthalmol. (2019) 33:260267. doi: 10.1016/j.sjopt.2019.07.010

5. Gass JDM. Focal congenital anomalies of the retinal pigment epithelium. Eye. (1989) 3:1-8. doi: 10.1038/eye.1989.2

6. Roseman RL, Gass JDM. Solitary hypopigmented nevus of the retinal pigment epithelium in the macula. Arch Ophthalmol. (1992) 110:1358-9. doi: 10.1001/archopht.1992.010802200 20005 
7. Su Y, Gurwood AS. Neurosensory retinal detachment secondary to torpedo maculopathy. Optometry. (2010) 81:405-7. doi: 10.1016/j.optm.2010.06.001

8. Bedar MS, Holz FG, Lischka T. Hypopigmentierung temporal der Makula. Fallvorstellung: Torpedo-Makulopathie. Der Ophthalmologe. (2013) 110:1734. doi: 10.1007/s00347-012-2705-x

9. Wong EN, Fraser-Bell S, Hunyor AP, Chen FK. Novel optical coherence tomography classification of torpedo maculopathy. Clin Experim Ophthalmol. (2015) 43:342-8. doi: 10.1111/ceo.12435

10. Pilotto E, Zannin ME, Convento E, Cortese M, Midena E. Torpedo maculopathy: a morphofunctional evaluation. Int Ophthalmol. (2013) 33:714. doi: 10.1007/s10792-012-9618-1

11. Hamm C, Shechtman D, Reynolds S. A deeper look at torpedo maculopathy. Clin Exp Optom. (2017) 100:563-8. doi: 10.1111/cxo.12540

12. Buzzonetti L, Petroni S, Catena G, Iarossi G. Optical coherence tomography and electrophysiological findings in torpedo maculopathy. Doc Ophthalmol. (2015) 130:65-70. doi: 10.1007/s10633-014-9472-8

13. Barbazetto IA, Maris PJG, Greenstein VC. Solitary albinotic spot of the retinal pigment epithelium: a functional and imaging study. Klin Monbl Augenheilkd. (2008) 225:295-297. doi: 10.1055/s-2008-1027236

14. Jurjevic D, Böni C, Barthelmes D, Fasler K, Becker M, Michels S, et al. Torpedo maculopathy associated with choroidal neovascularization. Klin Monbl Augenheilkd. (2017) 234:508-14. doi: 10.1055/s-0043100230

15. Grimaldi G, Scupola A, Sammarco MG, Marullo M, Blasi MA. Morphofunctional evaluation of torpedo maculopathy with optical coherence tomography angiography and microperimetry. Am J Ophthalmol Case Rep. (2018) 10:16568. doi: 10.1016/j.ajoc.2018.02.019

16. Papastefanou VP, Vázquez-Alfageme C, Keane PA, Sagoo MS. multimodality imaging of torpedo maculopathy with swept-source, en face optical coherence tomography and optical coherence tomography angiography. Retin Cases Brief Rep. (2018) 12:153-57. doi: 10.1097/ICB.0000000000000456

17. Giannakaki-Zimmermann H, Munk MR, Dysli C, Ebneter A, Wolf S, Zinkernagel MS. Optical coherence tomography angiography features of torpedo maculopathy. Retin Cases Brief Rep. (2019) 13:337-42. doi: 10.1097/ICB.0000000000000589

18. Ding Y, Yao B, Ye H, Yu Y. Multimodal imaging of torpedo maculopathy in a Chinese woman: a case report. BMC Ophthalmol. (2019) 19:154. doi: 10.1186/s12886-019-1161-7

19. Liang J, Williams DR, Miller DT. Supernormal vision and high-resolution retinal imaging through adaptive optics. J Opt Soc Am A. (1997) 14:288492. doi: 10.1364/JOSAA.14.002884

20. Roorda A, Romero-Borja F, Donnelly III WJ, Queener H, Hebert TJ, Campbell MCW. Adaptive optics scanning laser ophthalmoscopy. Opt Express. (2002) 10:405-12. doi: 10.1364/OE.10.000405

21. Hugo J, Beylerian M, Denion E, Aziz A, Gascon P, Denis D, et al. Multimodal imaging of torpedo maculopathy including adaptive optics. Eur J Ophthalmol. (2019) 30:NP27-31. doi: 10.1177/1120672119827772

22. Lambert NG, Grigorian F, Vasconcelos H, Watzke RC, Pennesi ME. (2020) Adaptive optics ophthalmoscopy, multifocal ERG and OCTA in unique case of suspected torpedo maculopathy presenting with vitelliform lesion. Am J Ophthalmol Case Rep. 20:100996. doi: 10.1016/j.ajoc.2020. 100996

23. Vienola KV, Zhang M, Snyder VC, Sahel JA, Dansingani KK, et al. Microstructure of the retinal pigment epithelium near-infrared autofluorescence in healthy young eyes and in patients with AMD. Sci Rep. (2020) 10:9561. doi: 10.1038/s41598-020-66581-x
24. Zhang M, Gofas-Salas E, Leonard BT, Rui Y, Snyder VC, Reecher HM, et al. Strip-based digital image registration for distortion minimization and robust eye motion measurement from scanned ophthalmic imaging systems. Biomed Opt Exp. (2021) 12:2353-2372. doi: 10.1364/BOE.418070

25. Rangel-Fonseca P, Gómez-Vieyra A, Malacara-Hernández D, Wilson MC, Williams DR, Rossi EA. Automated segmentation of retinal pigment epithelium cells in fluorescence adaptive optics images. J Opt Soc Am A. (2013) 30:2595-604. doi: 10.1364/JOSAA.30.002595

26. Laser Institute of America (2014). American National Standard for Safe Use of Lasers Z136.1.

27. Delori FC, Webb RH, Sliney DH. Maximum permissible exposures for ocular safety (ANSI 2000), with emphasis on ophthalmic devices. J Opt Soc Am A. (2007) 24:1250-65. doi: 10.1364/JOSAA.24.001250

28. Banaee T, Doss M, Eller AW. Torpedo maculopathy complicated by choroidal neovascularization. Am J Ophthalmol Case Rep. (2020) 19:100772. doi: 10.1016/j.ajoc.2020.100772

29. Granger CE, Yang Q, Song H, Saito K, Nozato K, Latchney LR, et al. Human retinal pigment epithelium: in vivo cell morphometry, multispectral autofluorescence, and relationship to cone mosaic. Invest Ophthalmol Vis Sci. (2018) 59:5705-16. doi: 10.1167/iovs.18-24677

30. Song H, Rossi EA, Yang Q, Granger CE, Latchney LR, Chung MM. High-resolution adaptive optics in vivo autofluorescence imaging in stargardt disease. JAMA Ophthalmol. (2019) 137:6039. doi: 10.1001/jamaophthalmol.2019.0299

31. Curcio CA, Sloan KR, Kalina RE, Hendrickson AE. Human photoreceptor topography. J Comparat Neurol. (1990) 292:497523. doi: 10.1002/cne.902920402

32. Light JG, Alvin Liu TY. A novel phenotype of torpedo maculopathy on spectral-domain optical coherence tomography. Am J Ophthalmol Case Rep. (2020) 20:100956. doi: 10.1016/j.ajoc.2020.100956

33. Dolz-Marco R, Saffra NA, Freund KB. Torpedo maculopathy presenting with a vitelliform lesion. Retina. (2017) 37:e19e20. doi: 10.1097/IAE.0000000000001305

Conflict of Interest: Some aspects of this work include technologies that ER is an inventor on for patents that are owned by the University of Rochester (US Patent No.: US 10,123,697 and US 10,092,181).

The remaining authors declare that the research was conducted in the absence of any commercial or financial relationships that could be construed as a potential conflict of interest.

Publisher's Note: All claims expressed in this article are solely those of the authors and do not necessarily represent those of their affiliated organizations, or those of the publisher, the editors and the reviewers. Any product that may be evaluated in this article, or claim that may be made by its manufacturer, is not guaranteed or endorsed by the publisher.

Copyright (C) 2021 Vienola, Dansingani, Eller, Martel, Snyder and Rossi. This is an open-access article distributed under the terms of the Creative Commons Attribution License (CC BY). The use, distribution or reproduction in other forums is permitted, provided the original author(s) and the copyright owner(s) are credited and that the original publication in this journal is cited, in accordance with accepted academic practice. No use, distribution or reproduction is permitted which does not comply with these terms. 